\title{
TWO-STEP PROCEDURES IN PALM THEORY
}

\author{
GERT NIEUWENHUIS \\ Tilburg University, Dept. of Econometrics \\ PO Box 90153, NL-5000 LE Tilburg, The Netherlands \\ E-mail: G.Nieuwenhuis@kub.nl
}

(Received July, 1998; Revised October, 2000)

\begin{abstract}
Random time changes (RTCs) are right-continuous and non-decreasing random functions passing the zero-level at 0 . The behavior of such systems can be studied from a randomly chosen time-point and from a randomly chosen level. From the first point of view, the probability characteristics are described by the time-stationary distribution $P$. From the second point of view, the detailed Palm distribution (DPD) is the ruling probability mechanism. The main topic of the present paper is a relationship between $P$ and its DPD. Under $P$, the origin falls in a continuous part of the graph. Under the DPD, there are two typical situations: the origin lies in a jump-part of the extended graph or it lies in a continuous part. These observations lead to two conditional DPDs. We derive two-step procedures, which bridge the gaps between the several distributions. One step concerns the application of a shift, the second is just a change of measure arranged by a weight-function. The procedures are used to derive local characterization results for the distributions of Palm type. We also consider simulation applications. For instance, a procedure is mentioned to generate a simulation of the RTC as seen from a randomly chosen level in a jump-part when starting with simulations from a randomly chosen timepoint. The point process with batch-arrivals is often used as an application.
\end{abstract}

Key words: Random Time Change, Palm Distribution, Detailed Palm Distribution, Local Characterization, Radon-Nikodym Derivative, Simulation Procedure.

AMS subject classifications: $60 \mathrm{G} 57,60 \mathrm{G} 55,60 \mathrm{G} 10,60 \mathrm{~K} 25$.

\section{Introduction}

Palm theory for queues and point process (PPs) studies a relationship between two probability mechanisms. Intuitively, the first one - the time-stationary distribution $P$ - describes the probability behavior of random phenomena within the (queueing) system as it is seen from a randomly chosen time-point. The second one - the Palm distribution $P^{0}$ - describes this behavior as it is seen from a randomly chosen 
occurrence (often an arriving customer). See, e.g., Slivnyak [16], Ryll-Nardzewski[13], Franken et al. [4], Baccelli and Brémaud [2], or Sigman [15] for a review. An important part of this Palm theory considers a direct relationship between the distributions $P$ and $P^{0}$ themselves. For instance, in Theorem 1.3.1 of [4] the local characterization of the Palm Distribution (shortly, PD) of a simple point process is formulated. Intuitively, this approximation theorem expresses that in a sense, $P^{0}$ can be considered as the conditional distribution of $P$ given an occurrence at the origin.

In Nieuwenhuis [11], it is proved that this local characterization is uniform and there the rate of convergence is studied. Also, a two-step procedure is given to transform $P$ into $P^{0}$ (or vice versa). (See also Thorisson [17] and Nieuwenhuis [12].) Starting from $P$, the first step is based on a shift, moving the origin to the last occurrence before it. It leads to a probability measure that gives exactly the same events zero probability as $P^{0}$. In the second step, this probability measure is transformed into $P^{0}$ by using Radon-Nikodym's theorem. The first step is based on a shift, the second one on a change of measure. This change of measure follows from Theorem 2.1 in Nieuwenhuis [10] where distributions are introduced which have the same nullsets as the PD and which can serve as a bridge between $P$ and $P^{0}$. As an immediate consequence of the simulation procedure in Section 14 of [17], this theorem gives an opportunity to obtain a simulation from $P^{0}$ when starting with simulations from the distribution $P$, in the case that, with probability one, the interarrival times are larger than a positive constant.

During recent years, the development of Palm theory has advanced. In Schmidt and Serfozo [14], an overview is given of Palm theory for random measures. In Miyazawa and Schmidt [8], Palm theory is applied in the context of risk processes, while Miyazawa [7] considers fluid queues. In Miyazawa et al. [9], a general view on Palm theory is presented by considering a random phenomenon of interest as a rightcontinuous and non-decreasing random function passing the zero-level at zero, i.e., as a random time change (RTC). This theory not only includes Palm theory for random measures, but also includes the theory for non-simple queueing systems and fluid queues with general jumps. For an RTC $\Lambda$, two distributions of Palm type are considered. The detailed Palm distribution (DPD) of $\Lambda$ is the most important one in the sense that, for instance, the ordinary Palm distribution (OPD) can immediately be obtained from it. Intuitively, the DPD of $\Lambda$ arises by another random-choosing procedure: choose a level at random on the vertical axis and shift the origin to the corresponding position on the extended graph of $\Lambda$ (i.e., the graph extended with the jump-parts).

In view of the advances of Palm theory for simple PPs via Palm theory for random measures to Palm theory for general RTCs, it is natural to study generalizations of specific results like the characterization theorem, the two-step procedure and the simulation procedure. Especially, these are interesting, because they will give a better understanding of rather new concepts like the DPD. In the present research, we will consider such generalizations for the DPD (and also the OPD) of RTCs. For the larger part, we will assume that the probability that the random function $\Lambda$ (or its generalized inverse) makes jumps is positive.

In Section 2, we will formulate the framework that is used. Some known results, needed for the present research, are recalled. Under the DPD, there are two typical types of realizations of the RTC. For the first type, the origin lies in a jump-part of the extended graph. For the second type, it lies in a continuous part. So, the DPD is a mixture of two conditional DPDs. In two lemmas, we study independencies under 
the several distributions. In Section 3, we derive several relations which express easytransitions from one distribution to another. Essentially, they express transformations by a two-step procedure. One step concerns a shift of the origin. The other step is based on Radon-Nikodym's theorem and just means a change of measure. Emphasis will be on the transition from $P$ to these two conditional DPDs. The topic of Section 4 is how to approximate one distribution under the regime of another. For instance, in the case that $P$ is the ruling distribution, we derive a local characterization of the conditional DPDs. The limit results turn out to hold uniformly. As an application, we also consider a local characterization of Palm probabilities in the case of a bulk arrival process. In Section 5, we apply the results of Sections 3 and 4 by considering simulation procedures. For instance, a version of the RTC as seen from a randomly chosen level in its jump-parts can be derived from versions as seen from a randomly chosen time-point. This procedure is of special interest when realizations of the RTC are difficult (or time-consuming) to obtain from the former point of view, but easier under the latter. If necessary, the resulting realization can be transformed into a realization of another desired Palm type (e.g., under OPD). Again, the results are applied for a point process with batch arrivals.

\section{Preliminaries}

Let $(\Omega, \mathcal{F})$ be a measurable space supporting the right-continuous, non-decreasing random function $\Lambda$ on $\mathbb{R}$ for which

$$
\lim _{t \rightarrow \pm \infty} \Lambda(t)= \pm \infty \text { and } \Lambda(0-) \leq 0 \leq \Lambda(0)
$$

for all $\omega \in \Omega$. We call $\Lambda$ a random time change, RTC for short. The extended graph $\Gamma(\omega)$ of $\Lambda(\cdot, \omega)$ is a subset of $\mathbb{R}^{2}$ consisting of the graph of $\Lambda(\cdot, \omega)$ supplied with the vertical jump-parts:

$$
\Gamma(\omega)=\left\{(t, x) \in \mathbb{R}^{2}: \Lambda(t-, \omega) \leq x \leq \Lambda(t, \omega)\right\} .
$$

In practice, often the canonical setting is considered. That is, $\Omega$ is taken as the set $G$ of all right-continuous and non-decreasing functions $g: \mathbb{R} \rightarrow \mathbb{R}$ with $\lim _{t \rightarrow \pm \infty} g(t)=$ $\pm \infty$, passing the zero-level at zero. We will, however, consider the general, abstract setup.

We will always assume that a family $\Theta=\left\{\Theta_{(t, x)}:(t, x) \in \mathbb{R}^{2}\right\}$ of transformations exists - possibly on a larger measurable space $(\tilde{\Omega}, \tilde{\mathscr{F}})$ - in such a way that the (random) extended graph $\Gamma$ of $\Lambda$ is consistent with $\Theta$, and that the family $\Theta$ behaves on $\Gamma$ as a group. I.e., we assume that:

(i) For all $\omega \in \Omega,(t, x) \in \Gamma(\omega)$ and $(s, y) \in \Gamma\left(\Theta_{(t, x)} \omega\right)$ we have

(a) $\quad \Lambda\left(\cdot, \Theta_{(t, x)} \omega\right)=\Lambda(t+\cdot, \omega)-x$,

(b) $\Theta_{(s, y)}\left(\Theta_{(t, x)}^{\omega)}=\Theta_{(s+t, x+y)^{\omega}}\right.$.

Note that, in the canonical setting, this assumption is trivially satisfied: just take $\tilde{\Omega}$ as the set $\widetilde{G}$ arising from $G$ by omitting the condition about passing the zero-level, and define $\Theta_{(t, x)} g$ by $g(t+\cdot)-x$. For details, we refer to [9].

The right-continuous inverse function $\Lambda^{\prime}(\cdot, \omega)$ is defined by

$$
\Lambda^{\prime}(x, \omega)=\sup \{s \in \mathbb{R}: \Lambda(s, \omega) \leq x\}, \quad x \in \mathbb{R} .
$$

Hence, $\Lambda^{\prime}$ is another RTC. From the two-dimensional family $\Theta$, we define two one- 
dimensional groups, $\left\{\theta_{t}: t \in \mathbb{R}\right\}$ and $\left\{\eta_{x}: x \in \mathbb{R}\right\}$, of transformations by

$$
\theta_{t} \omega:=\Theta_{(t, \Lambda(t, \omega))} \omega \text { and } \eta_{x} \omega=\Theta_{\left(\Lambda^{\prime}(x, \omega), x\right)} \omega, \omega \in \Omega \text { and } t, x \in \mathbb{R} \text {. }
$$

It follows immediately that, for all $\omega \in \Omega$ and $t, s, x, y \in \mathbb{R}$,

$$
\begin{aligned}
& \Lambda(t) \circ \theta_{s}=\Lambda(t+s)-\Lambda(s), \\
& \Lambda^{\prime}(y) \circ \eta_{x}=\Lambda^{\prime}(y+x)-\Lambda^{\prime}(x), \\
& \Lambda^{\prime}(x) \circ \theta_{t}=\Lambda^{\prime}(x,+\Lambda(t))-t, \\
& \Lambda(t) \circ \eta_{x}=\Lambda\left(t+\Lambda^{\prime}(x)\right)-x, \\
& \eta_{x} \circ \theta_{t}=\eta_{x}+\Lambda(t) \text { and } \theta_{t} \circ \eta_{x}=\theta_{t+\Lambda^{\prime}(x)} .
\end{aligned}
$$

The transformations $\theta_{t}$ and $\eta_{x}$ will be called shifts. By Lemma 2.3 of the above reference, the two invariant $\sigma$-fields of the $\theta$-shifts and the $\eta$-shifts coincide. We denote this common $\sigma$-field by $\mathrm{g}$ and note that $\mathrm{g}$-measurable functions on $\Omega$ are invariant under all shifts of both types.

We next introduce a probability measure $P$ on $(\Omega, \mathcal{F})$. We will always assume that under $P$, the $\theta$-group is stationary and the long-run average $\bar{\Lambda}:=\lim _{t \rightarrow \infty} \Lambda(t) / t$ is positive and finite, i.e.,

(ii) $\quad P\left(\theta_{t}^{-1} A\right)=P(A)$ for all $t \in \mathbb{R}$ and $A \in \mathscr{F}$,

(iii) $\quad P(0<\bar{\Lambda}<\infty)=1$.

So, we assume that the RTC $\Lambda$ satisfies $(i)-(i i i)$. These assumptions are sufficient to consider the probability measure $P_{\Lambda}$, the detailed Palm distribution (DPD) of $P$ w.r.t. $\Lambda$. It is defined by

$$
P_{\Lambda}(A)=E\left(\frac{1}{\bar{\Lambda}} \int_{0}^{\Lambda(1)} 1_{A} \circ \eta_{x} d x\right), A \in \mathcal{F} .
$$

In [9], it is proved that all shifts $\eta_{y}, y \in \mathbb{R}$, are measure preserving under $P_{\Lambda}$. So, the $\eta$-group is stationary under $P_{\Lambda}$. It is also proved that the following inversion formula holds:

$$
P(A)=E_{\Lambda}\left(\frac{1}{\bar{\Lambda}^{\prime}} \int_{0}^{\Lambda^{\prime}(1)} 1_{A} \circ \theta_{s} d s\right), A \in \mathcal{F},
$$

and that $P_{\Lambda}$ and $P$ are dual in the sense that the DPD of $P_{\Lambda}$ w.r.t. $\Lambda^{\prime}$ returns $P$. Here $\bar{\Lambda}^{\prime}:=\lim _{t \rightarrow \infty} \Lambda^{\prime}(t) / t=1 / \bar{\Lambda}$, and $E_{\Lambda}$ denotes expectation under $P_{\Lambda}$. Another distribution of Palm type is also considered: the well-known (ordinary) Palm distribution $P^{0}$ (shortly OPD) of $P$ w.r.t. $\Lambda$. It is defined below with the right-hand side as the most known:

$$
P^{0}(A)=E\left(\frac{1}{\bar{\Lambda}} \int_{0}^{\Lambda(1)} 1_{A} \circ \theta_{\Lambda^{\prime}(x)} d x\right)=E\left(\frac{1}{\bar{\Lambda}} \int_{(0,1]} 1_{A} \circ \theta_{t} \Lambda(d t)\right), \quad A \in \mathscr{F} .
$$

The right-hand side is a natural extension of the case where $\Lambda$ is a random counting measure and is essentially due to Mecke [6]. From the left-hand side of (2.4) and the stationarity property of $P_{\Lambda}$, it follows that 
DPD and OPD coincide iff $P(\Lambda$ is continuous $)=1$ iff $P_{\Lambda}(\Lambda(0)=0)=1$.

In a sense, it was already noted in Geman and Horowitz [5] that a duality between $P$ and $P^{0}$ appears in the continuous case. Hence, the DPD can be viewed as the distribution of Palm type which restores duality also in the non-continuous case. It turns out that the DPD is a very natural distribution (see (2.5)-(2.8) below), which behaves like "the mother of all PDs" in the sense that a PD needed in a specific context can immediately be deduced from it. See [9] for more details and Example 3.1 below for the deduction of a PD with nice stationarity properties in the case of a point process with possibly non-single occurrences.

The relationship between $P, P_{\Lambda}$, and $P^{0}$ is expressed further in the following formulas. Here $E^{0}$ denotes the expectation under $P^{0}$, and $\Lambda\{0\}:=\Lambda(0)-\Lambda(0-)$ is the jump-size at 0 . The expressions in (2.6) hold both $P$ - and $P_{\Lambda^{-a . s:}}$

$$
\begin{gathered}
P^{0}(A)=P_{\Lambda}\left(\theta_{0}^{-1} A\right) \quad \text { and } \quad P_{\Lambda}(A)=E^{0}\left(\frac{1}{\Lambda\{0\}} \int_{-\Lambda\{0\}}^{0} 1_{A} \circ \eta_{x} d x\right) \\
\lim _{t \rightarrow \infty} \frac{1}{t} \int_{0}^{t} 1_{A} \circ \theta_{s} d s=P(A \mid g) \quad \text { and } \lim _{y \rightarrow \infty} \frac{1}{y} \int_{0}^{y} 1_{A^{\circ}} \circ \eta_{x} d x=P_{\Lambda}(A \mid g), \\
\lim _{y \rightarrow \infty} \frac{1}{y} \int_{0}^{y} P\left(\eta_{x}^{-1} A\right) d x=P_{\Lambda}(A) \text { and } \lim _{y \rightarrow \infty} \frac{1}{y} \int_{0}^{y} P\left(\theta_{\Lambda^{\prime}(x)}^{-1} A\right) d x=P^{0}(A), \\
\lim _{t \rightarrow \infty} \frac{1}{t} \int_{0}^{t} P_{\Lambda}\left(\theta_{s}^{-1} A\right) d s=P(A) ; A \in \mathscr{F} .
\end{gathered}
$$

(The averaged integral in (2.5) is interpreted as $1_{A}(\omega)$ if $\Lambda(\{0\}, \omega)=0$.) These relations have very natural and intuitive meanings in the canonical case. For instance, the left-hand side of (2.7) indicates that, starting with $P$, we can obtain $P_{\Lambda}$ by randomly choosing a level $x$ on the (vertical) positive half-line and shifting the origin to the position on the extended graph which corresponds to this level. The OPD can be obtained by shifting (if necessary) this randomly chosen position $x$ up to the closest position on the graph. For more details, again we refer to [9]. In this reference, it is also proven that every random measure $\Lambda^{*}$, which satisfies the usual stationarity assumptions in random measure theory, can be characterized by a suitably chosen RTC $\Lambda$ satisfying (i)-(iii). Hence, this RTC setting includes the random measure framework. Essentially, it is a straightforward generalization of the framework in [5]. In this reference, it is additionally assumed that $\Lambda(0)=0$ for all $\omega \in \Omega$ (and for many of the results even that $\Lambda$ is continuous), leading to a setting which is not suitable to support the concept of a DPD.

Since so many distributions are involved, we will sometimes attach the distribution to describe a property. For instance, we will talk about $P$-independency if independency under $P$ is meant. $P_{\Lambda}$-distributed means distributed under $P_{\Lambda}$.

If $\Lambda$ is continuous and strictly increasing (with probability one), then both $\Lambda$ and $\Lambda^{\prime}$ makes no jumps. This case will be shortly considered now, under the assumption 
that, with probability one, $\Lambda$ is almost everywhere differentiable. At first, note that $P_{\Lambda}$ and $P^{0}$ coincide because of continuity. Writing $\lambda(\cdot, \omega)$ for the derivative of $\Lambda(\cdot, \omega)$ it follows immediately from the assumptions that, at least $P$-a.s.,

$$
\lambda(t, \omega)=\lambda\left(0, \theta_{t} \omega\right) \text { for almost all } t \in \mathbb{R} .
$$

From the right-hand side of (2.4) we obtain that

$$
P_{\Lambda}(A)=P^{0}(A)=E\left(\frac{\lambda(0)}{\bar{\Lambda}} 1_{A}\right), \quad A \in \mathcal{F} .
$$

So $P_{\Lambda}$ arises from $P$ by changing the importance of the realizations by way of the weight function $\lambda(0) / \bar{\Lambda}$. That is, $P_{\Lambda}$ describes a change of measure.

The case that $\Lambda$ makes jumps is more complicated. For the larger part of the present paper we will assume that $\Lambda$ makes jumps, i.e., that

(iv) $\quad p:=P_{\Lambda}(\Lambda\{0\}>0)$ is positive.

The case that $\Lambda$ is continuous (that is, $p=0$ ) while its graph has horizontal parts, implies that $\Lambda^{\prime}$ makes jumps. This case can be treated by using the duality property mentioned above and will be considered shortly in Section 3.

Assume that $(i v)$ is satisfied. Let $T_{i}, i \in \mathbb{Z}$, be the (random) jump times at which the RTC jumps, under the convention that $\ldots<T_{-1}<T_{0} \leq 0<T_{1}<T_{2}<\ldots$ Furthermore, set $D_{i}:=\Lambda\left(T_{i}-\right)$ and $S_{i}:=\Lambda\left(T_{i}\right), i \in \mathbb{Z}$. If $q:=1-p=P_{\Lambda}(\Lambda\{0\}=$ 0 ) is also positive, then there are, under $P_{\Lambda}$, two typical types of realizations. The important point here is the position of the origin: it falls in a jump-part of the extended graph, or in a continuous part. Set

$$
P_{1 \Lambda}:=P_{\Lambda}(\cdot \mid \Lambda\{0\}>0) \text { and } P_{2 \Lambda}:=P_{\Lambda}(\cdot \mid \Lambda\{0\}=0) .
$$

The two types of realizations are just versions of the RTC under $P_{1 \Lambda}$ and $P_{2 \Lambda}$, respectively. Note that

$$
P_{\Lambda}=p P_{1 \Lambda}+q P_{2 \Lambda}
$$

It is not forbidden that $D_{i}-S_{i-1}$ equals zero, i.e., that $\Lambda$ does not increase on $\left(T_{i-1}, T_{i}\right)$. We want to identify the non-empty intervals $\left(S_{i-1}, D_{i}\right)$ on the vertical axis. For $i \in \mathbb{Z}$, let $K(i)$ be the index of the $i$ th jump-time in order for which $D_{K(i)+1}-S_{K(i)}$ is strictly positive, with the convention that $K(1) \geq 1$ and $K(0) \leq 0$. Set $T_{i}^{*}:=T_{K(i)}, S_{i}^{*}:=\Lambda\left(T_{i}^{*}\right)$ and $D_{i+1}^{*}:=\Lambda\left(T_{K(i)+1}-\right)$ Note that $D_{i+1}^{*}$ is not necessarily equal to $\Lambda\left(T_{i+1}^{*}-\right)$, and that $D_{i+1}^{*}-S_{i}^{*}>0$. Furthermore, set

$$
U: \frac{T_{1}}{T_{1}-T_{0}}, U^{*}:=\frac{T_{1}^{*}}{T_{1}^{*}-T_{0}^{*}}, U^{\prime}:=\frac{S_{0}}{S_{0}-D_{0}} \text { and } U^{\prime \prime}:=\frac{D_{1}^{*}}{D_{1}^{*}-S_{0}^{*}} .
$$

(The definitions of $U^{*}$ and $U^{\prime \prime}$ require that $q>0$.) Note that $U^{\prime \prime}=D_{1} /\left(D_{1}-S_{0}\right)$ $P_{2 \Lambda^{-}}$a.s. since $P_{\Lambda}(\Lambda(t)>0$ for all $t>0)=1$ by $(2.2)$.

Lemma 2.1: Assume that $\Lambda$ satisfies $(i)-(i v)$. Then:

(a) For all $t \in \mathbb{R}$, it holds that $\theta_{t}^{-1_{\mathcal{F}}}$ and $U^{\prime}$ are independent under $P_{1 \Lambda}$, while $U^{\prime}$ is uniformly $(0,1)$ distributed.

(b) For all $n \in \mathbb{Z}$, it holds that $\theta_{T}^{-1}{ }^{1} \mathcal{F}$ and $U$ are independent under $P$, while $U$ is uniformly $(0,1)$ distributed. 
(c) Let $q>0$. For all $n \in \mathbb{Z}$, it holds that $\theta_{T_{n}^{*}}^{-1} \mathscr{F}$ and $U^{*}$ are independent under $P$, while $U^{*}$ is uniformly $(0,1)$ distributed.

(d) Let $q>0$. For all $n \in \mathbb{Z}$, it holds that $\theta_{T_{n}^{-1}}^{-1} \mathcal{F}$ and $U^{\prime \prime}$ are independent under $P_{2 \Lambda}$, while $U^{\prime \prime}$ is uniformly $(0,1)$ distributed.

Proof: Let $f$ and $g$ be suitably measurable functions. Denote the number of $S_{i} \mathrm{~s}$ in $(0, y]$ by $M(y)$. By $(2.7)$ and $(2.1)$, we have, since $\Lambda^{\prime}(x)=T_{i}$ for all $x \in\left(D_{i}, S_{i}\right)$,

$$
\begin{gathered}
E_{1 \Lambda}\left(f\left(U^{\prime}\right) g \circ \theta_{t}\right)=\frac{1}{p} \lim _{y \rightarrow \infty} E\left(\frac{1}{y} \int_{0}^{y} f\left(U^{\prime} \circ \eta_{x}\right) \cdot g \circ \theta_{t} \circ \eta_{x} \cdot 1_{(\Lambda\{0\}>0)} \circ \eta_{x} d x\right) \\
=\frac{1}{p} \lim _{y \rightarrow \infty} E\left(\frac{1}{y} \sum_{i=1}^{M(y)} \int_{D_{i}}^{S_{i}} f\left(\frac{S_{i}-x}{S_{i}-D_{i}}\right) d x \cdot g \circ \theta_{t+T_{i}}\right) \\
=\frac{1}{p} \int_{0}^{1} f(x) d x \cdot \lim _{y \rightarrow \infty} E\left(\frac{1}{y} \sum_{i=1}^{M(y)}\left(g \circ \theta_{t+T_{i}}\left(S_{i}-D_{i}\right)\right)\right) .
\end{gathered}
$$

Applying the above for $f \equiv 1$, yields for general $f$ and $g$ :

$$
E_{1 \Lambda}\left(f\left(U^{\prime}\right) g \circ \theta_{t}\right)=\int_{0}^{1} f(x) d x \cdot E_{1 \Lambda}\left(g \circ \theta_{t}\right) .
$$

Part $(a)$ follows. Parts $(b)$ and $(c)$ can be proven in a similar way by using $(2.8)$ and partitioning $(0, t]$ into the parts $\left(T_{i-1}, T_{i}\right]$ and $\left(T_{i-1}^{*}, T_{i}^{*}\right]$, respectively. For $(d)$, we use (2.7) again and partition $(0, y]$ into the intervals $\left(S_{i-1}, D_{i}\right]$; note that $U^{\prime \prime}=$ $D_{1} /\left(D_{1}-S_{0}\right)$ under $P_{2 \Lambda}$.

We need more definitions. Set

$$
\begin{gathered}
\gamma_{i-1}:=\frac{T_{i}-T_{i-1}}{S_{i-1}-D_{i-1}} \bar{\Lambda} \quad \text { and } \quad \delta_{i-1}:=\frac{T_{i}^{*}-T_{i-1}^{*}}{D_{i}^{*}-S_{i-1}^{*}} \bar{\Lambda}, i \in \mathbb{Z} \\
X:=U \gamma_{0}=\frac{T_{1}}{S_{0}-D_{0}} \bar{\Lambda} \quad \text { and } Y:=U^{*} \delta_{0}=\frac{T_{i}^{*}}{D_{1}^{*}-S_{0}^{*}} \bar{\Lambda}
\end{gathered}
$$

provided that these random variables are well defined. (For instance, the definition of $\delta_{i-1}$ requires that both $p$ and $q$ are positive.)

Lemma 2.2: Assume that $\Lambda$ satisfies $(i)-(i v)$. Then:

(a) $U$ and $\gamma_{0}$ are $P$-independent. If $q>0$, then $U^{*}$ and $\delta_{0}$ are $P$-independent.

(b) $\quad U^{\prime}$ and $\gamma_{0}$ are $P_{1 \Lambda}$-independent.

(c) If $q>0$, then $U^{\prime \prime}$ and $\delta_{0}$ are $P_{2 \Lambda}$-independent.

(d) The conditional $P$-distribution of $X$, given $\gamma_{0}$, is uniform on $\left(0, \gamma_{0}\right)$.

(e) If $q>0$, then the conditional P-distribution of $Y$, given $\delta_{0}$, is uniform on $\left(0, \delta_{0}\right)$. 
Proof: Since $\gamma_{0}=\gamma_{0} \circ \theta_{T_{0}}, \delta_{0}=\delta_{0} \circ \theta_{T_{0}^{*}}$, and $\gamma_{0}=\gamma_{0} \circ \theta_{0}$, parts $(a),(b)$ and $(c)$ follow immediately from Lemma 2.1. For nonnegative $a$ and $b$, we obtain, by conditioning on $\gamma_{0}$, that

$$
P\left(\gamma_{0} \leq a \text { and } X \leq b\right)=E\left(1_{\left(\gamma_{0} \leq a\right)}\left(1 \wedge \frac{b}{\gamma_{0}}\right)\right)
$$

cf. (2.11) and (a). A similar result holds for $\delta_{0}$ and $Y$. Parts $(d)$ and $(e)$ follow immediately.

\section{Two-Step Procedures}

At first sight, there seem to be large gaps between the distribution $P$ on one hand and the distributions of Palm type on the other. However, the gaps can be bridged by simple two-step procedures. For the PP case with single occurrences, it is wellknown that the time-stationary distribution $P$ can be transformed into the PD by a two-step procedure. First, the origin is shifted to the last arrival on its left, then, a change of measure is applied. In this section, we will study similar bridging procedures for the general RTC case.

In view of Lemma 2.1 , we can, in a sense, consider

$$
\sigma:=D_{0}+U\left(S_{0}-D_{0}\right) \text { and } \tau:=S_{0}^{*}+U^{*}\left(D_{1}^{*}-S_{0}^{*}\right)
$$

as arbitrarily chosen positions in the intervals $\left(D_{0}, S_{0}\right)$ and $\left(S_{0}^{*}, D_{1}^{*}\right)$, respectively. With this observation in mind, the following theorem is less surprising.

Theorem 3.1: Let $\Lambda$ be an RTC which satisfies $(i)-($ iii).

(a) If $p>0$, then $P\left(\eta_{\sigma}^{-1} A\right)=p E_{1 \Lambda}\left(\gamma_{0} 1_{A}\right), A \in \mathcal{F}$.

(b) If $p, q>0$, then $P\left(\eta_{\tau}^{-1} A\right)=q E_{2 \Lambda}\left(\delta_{0} 1_{A}\right), A \in \mathscr{F}$.

Proof: (a) The proof is mainly based on (2.6) and (2.8). Let the number of jump times in the interval $(0, t]$ be denoted by $N(t)$. Since

$$
\eta_{\sigma} \circ \theta_{s}=\eta_{L(s)} \text { with } L(s):=D_{i-1}+\frac{T_{i}-s}{T_{i}-T_{i-1}}\left(S_{i-1}-D_{i-1}\right),
$$

for all $\omega \in \Omega$ and $s$ between $T_{i-1}(\omega)$ and $T_{i}(\omega)$, we obtain (by decomposing the integral and changing the variable of integration):

$$
\begin{gathered}
P\left(\eta_{\sigma}^{-1} A\right)=\lim _{t \rightarrow \infty} E_{\Lambda}\left(\frac{1}{t} \sum_{i=1}^{N(t)} \int_{T_{i-1}}^{T_{i}} 1_{A} \circ \eta_{\sigma} \circ \theta_{s} d s\right) \\
=\lim _{t \rightarrow \infty} E_{\Lambda}\left(\frac{1}{t} \sum_{i=1}^{N(t)} \int_{D_{i-1}}^{S_{i-1}} 1_{A} \circ \eta_{x} \cdot \frac{T_{i}-T_{i-1}}{S_{i-1}-D_{i-1}} d x\right) \\
=\lim _{t \rightarrow \infty} E_{\Lambda}\left(\frac{\Lambda(t)}{t} \frac{1}{\Lambda(t)} \int_{0}^{\Lambda(t)} 1_{A}^{\circ} \eta_{x} \cdot \frac{\left(T_{1}-T_{0}\right) \circ \eta_{x}}{\left(S_{0}-D_{0}\right) \circ \eta_{x}} 1_{(\Lambda\{0\}>0)} \circ \eta_{x} d x\right)
\end{gathered}
$$




$$
=E_{\Lambda}\left(\bar{\Lambda} E_{\Lambda}\left(1_{A} \cdot \frac{T_{1}-T_{0}}{S_{0}-D_{0}} \cdot 1_{(\Lambda\{0\}>0)} \mid g\right)\right)=p E_{1 \Lambda}\left(\gamma_{0} 1_{A}\right)
$$

The proof of $(b)$ is similar. Let $N^{*}(t)$ be the number of $T_{i}^{*} \mathrm{~s}$ in $(0, t]$. Then,

$$
\begin{gathered}
P\left(\eta_{\tau}^{-1} A\right)=\lim _{t \rightarrow \infty} E_{\Lambda}\left(\frac{1}{t} \sum_{i=1}^{N^{*}(t)} \int_{T_{i-1}^{*}}^{T_{i}^{*}} 1_{A} \circ \eta_{\tau} \circ \theta_{s} d s\right) \\
=\lim _{t \rightarrow \infty} E_{\Lambda}\left(\frac{1}{t} \sum_{i=1}^{N^{*}(t)}\left(\int_{S_{i-1}^{*}}^{D_{i}^{*}} 1_{A} \circ \eta_{x} d x \cdot \frac{T_{i}^{*}-T_{i-1}^{*}}{D_{i}^{*}-S_{i-1}^{*}}\right)\right) \\
=\lim _{t \rightarrow \infty} E_{\Lambda}\left(\frac{\Lambda(t)}{t} \frac{1}{\Lambda(t)} \int_{0}^{\Lambda(t)} 1_{A}^{\circ} \eta_{x} \cdot \frac{\left(T_{1}^{*}-T_{0}^{*}\right) \circ \eta_{x}}{\left(D_{1}^{*}-S_{0}^{*}\right) \circ \eta_{x}}(\Lambda\{0\}=0) \circ \eta_{x} d x\right) \\
=E_{\Lambda}\left(\overline { \Lambda } E _ { \Lambda } \left(1_{A} \cdot \frac{T_{1}^{*}-T_{0}^{*}}{\left.\left.D_{1}^{*}-S_{0}^{*} 1_{(\Lambda\{0\}=0)} \mid g\right)\right)=q E_{2 \Lambda}\left(\delta_{0} 1_{A}\right) .}\right.\right.
\end{gathered}
$$

As a consequence of part $(a)$, the distributions $P_{1 \Lambda}$ and $P \eta_{\sigma}^{-1}$ have the same null sets. If a statement holds $P_{1 \Lambda^{-a}}$.s. then it holds $P \eta_{\sigma}^{-1}$-a.s. and vice versa. The transformation of $P_{1 \Lambda}$ into $P \eta_{\sigma}^{-1}$, as expressed in part $(a)$, is nothing else but a change of measure. In order to arrange this, the weight function $p \gamma_{0}$, i.e., the RadonNikodym density of $P \eta_{\sigma}^{-1}$ w.r.t. $P_{1 \Lambda}$, is used to transform $P_{1 \Lambda}$ into $P \eta_{\sigma}^{-1}$. Similar remarks can be made for part $(b)$.

Since $\gamma_{0}$ and $\delta_{0}$ are invariant under the shifts $\eta_{\sigma}$ and $\eta_{\tau}$, we have

$$
\begin{gathered}
P_{1 \Lambda}(A)=\frac{1}{p} E\left(\frac{1}{\gamma_{0}} 1_{A} \circ \eta_{\sigma}\right) \text { and } P_{2 \Lambda}(A)=\frac{1}{q} E\left(\frac{1}{\delta_{0}} 1_{A} \circ \eta_{\tau}\right) \\
P_{\Lambda}(A)=E\left(\frac{1}{\gamma_{0}} 1_{A} \circ \eta_{\sigma}\right)+E\left(\frac{1}{\delta_{0}} 1_{A} \circ \eta_{\tau}\right), A \in \mathcal{F}
\end{gathered}
$$

if $p$ and $q$ are positive. Consequently,

$$
E\left(\frac{1}{\gamma_{0}}\right)=p=\frac{1}{E_{1 \Lambda}\left(\gamma_{0}\right)} \text { and } E\left(\frac{1}{\delta_{0}}\right)=q=\frac{1}{E_{2 \Lambda}\left(\delta_{0}\right)}
$$

The fractions of the increase of the random function $\Lambda$ caused by jumps and continuous parts are just the fractions $p$ and $q$, respectively.

Recall the definitions of $U^{\prime}$ and $U^{\prime \prime}$ below (2.9). Set $V:=T_{0}+U^{\prime}\left(T_{1}-T_{0}\right)$ and $W:=T_{0}^{*}+U^{\prime \prime}\left(T_{1}^{*}-T_{0}^{*}\right)$. In a sense, $\eta_{\sigma}$ and $\theta_{V}$ (and also $\eta_{\tau}$ and $\theta_{W}$ ) are inverse transformations. This is expressed in the following relations:

$$
\theta_{V} \circ \eta_{\sigma}=\theta_{0} \text { and } \eta_{\sigma} \circ \theta_{V}=\eta_{0} P_{1 \Lambda^{- \text {a.s. }}}
$$




$$
\begin{aligned}
& \theta_{W} \circ \eta_{\tau}=\theta_{0} \text { and } \eta_{\tau} \circ \theta_{W}=\eta_{0} P_{2 \Lambda^{-}} \text {a.s., } \\
& \eta_{\sigma} \circ \theta_{W}=\eta_{D_{0}-\left(S_{0} /\left(D_{1}-S_{0}\right)\right)\left(S_{0}-D_{0}\right)} \text { if } T_{0}^{*}=T_{0} \text { and } T_{1}^{*}=T_{1}, \\
& \eta_{\tau} \circ \theta_{V}=\eta_{S_{0}-\left(D_{0} /\left(S_{0}-D_{0}\right)\right)\left(D_{1}-S_{0}\right)} \text { if } T_{0}^{*}=T_{0} \text { and } T_{1}^{*}=T_{1} .
\end{aligned}
$$

As a consequence of Theorem 3.1, and (3.4) and (3.5) we obtain, for all $A \in \mathcal{F}$ :

$$
\begin{gathered}
P(A)=p E_{1 \Lambda}\left(\gamma_{0} 1_{A} \circ \theta_{V}\right) \text { if } p>0, \\
P(A)=q E_{2 \Lambda}\left(\delta_{0} 1_{A} \circ \theta_{W}\right) \text { if } p, q>0 .
\end{gathered}
$$

Theorem 3.1 and relations (3.2), (3.8) and (3.9) can be summarized in the following diagrams, where $\sim$ means that the corresponding probability measures have the same null-sets.

$$
\begin{array}{rlrl}
P & \sim P_{1 \Lambda} \theta_{V}^{-1} & P & \sim P_{2 \Lambda} \theta_{W}^{-1} \\
\eta_{\sigma} \downarrow & \downarrow \eta_{\sigma} & \eta_{\tau} \downarrow & \downarrow \eta_{\tau} \\
P \eta_{\sigma}^{-1} & \sim P_{1 \Lambda} & P \eta_{\tau}^{-1} & \sim P_{2 \Lambda}
\end{array}
$$

The left-hand diagram expresses that the Radon-Nikodym density of $P$ w.r.t. $P_{1 \Lambda} \theta_{V}^{-1}$, i.e., $p \gamma_{0}$ is not affected by applying the shift $\eta_{\sigma}$ (cf. Theorem $3.1(a)$ and (3.8)). Transformation of $P$ into $P_{1 \Lambda}$ (or vice versa) can be arranged by either of two two-step procedures. The first procedures goes clockwise, with the first step being a change of measure and the second one, the application of the shift $\eta_{\sigma}$ (or $\theta_{V}$ ). The second two-step procedure works counter-clockwise with the steps in the reversed order. For the right-hand diagram similar observations hold. See also, p. 47 in [11] for the PD in the single (simple) point process case, and Sections 4 and 5 in [17].

For completeness, we note that the above results can (if $p, q>0$ ) also be used to transform $P_{2 \Lambda}$ into $P_{1 \Lambda}$ or vice versa. The application of (3.2) and (3.9), and (3.2) and (3.8), respectively, yield:

$$
\begin{gathered}
P_{1 \Lambda}(A)=\frac{q}{p} E_{2 \Lambda}\left(\frac{\delta_{0}}{\gamma_{0} \circ \theta_{W}} 1_{A} \circ \eta_{\sigma} \circ \theta_{W}\right), \\
P_{2 \Lambda}(A)=\frac{p}{q} E_{1 \Lambda}\left(\frac{\gamma_{0}}{\delta_{0}} 1_{A} \circ \eta_{\tau} \circ \theta_{V}\right) ; \quad A \in \mathcal{F} .
\end{gathered}
$$

See also (3.6) and (3.7).

We next investigate corollaries for $P^{0}$, the OPD of $\Lambda$. Set

$$
P_{1}^{0}:=P^{0}(\cdot \mid \Lambda\{0\}>0) \text { and } P_{2}^{0}:=P^{0}(\cdot \mid \Lambda\{0\}=0) .
$$

If $p=0$ (that is, if $\Lambda$ is continuous), then $P_{\Lambda}=P_{2 \Lambda}=P^{0}=P_{2}^{0}$. If $p=1$ (i.e., if $\Lambda$ is a step-function, a pure jump process), then $P_{\Lambda}=P_{1 \Lambda}$ and $P^{0}=P_{1}^{0}=P_{\Lambda} \theta_{0}^{-1}$. In the case that $0<p<1$, it follows by $(2.5)$ that

$$
P^{0}=p P_{1}^{0}+q P_{2}^{0}, \quad P_{1}^{0}=P_{1 \Lambda} \theta_{0}^{-1} \text { and } P_{2}^{0}=P_{2 \Lambda} .
$$

Consequently, the above right-hand diagram can also be used for $P_{2}^{0}$. For $P_{1}^{0}$, it follows from (3.13), (3.2), (3.8) and (2.5) that 


$$
P_{1}^{0}(A)=\frac{1}{p} E\left(\frac{1}{\gamma_{0}} 1_{A} \circ \theta_{T_{0}}\right) \text { and } P(A)=p E_{1}^{0}\left(\frac{\bar{\Lambda}}{\Lambda\{0\}} \int_{0}^{T_{1}} 1_{A} \circ \theta_{s} d s\right)
$$

$A \in$ F. Here $E_{1}^{0}$ denotes the expectation under $P_{1}^{0}$. With $P^{\prime}$ defined by

$$
P^{\prime}(A):=\frac{1}{p} E\left(\frac{1}{\gamma_{0}} 1_{A}\right), A \in \mathcal{F}
$$

we obtain the following diagram:

$$
\begin{aligned}
P & \sim P^{\prime} \\
\theta_{T_{0}} \downarrow & \stackrel{\downarrow}{ } \theta_{T_{0}} \\
P \theta_{T_{0}}^{-1} & \sim P_{1}^{0}
\end{aligned}
$$

Similar to the diagrams in (3.10), this diagram gives two two-step procedures to generate $P_{1}^{0}$ from $P$, clockwise or counter-clockwise. In fact, this is the immediate generalization of the diagram in [11]. See also [17].

Example 3.1: Let $\Lambda=\Phi$ be a point process (i.e., an RTC with $p=1$ and integervalued jump-sizes), which satisfies $(i)$-(iii). The relationships between $P$, the DPD, and the OPD follow immediately from the above theory. However, in [9] it was noted that in this point process setting, a modification of the DPD is desirable since now only discrete positions (representing customers, for example) within the jumpparts are of interest. The following distribution incorporates this:

$$
\bar{P}_{\Phi}:=P_{\Phi} \eta_{\alpha}^{-1}
$$

with $\alpha:=\max \left\{\Phi(0)-i: i \in \mathbb{N}_{0}\right.$ and $\left.\Phi(0)-i \leq 0\right\}$ being the largest non-positive number which is integer-distanced from $\Phi(0)$. The distribution $\bar{P}_{\Phi}$ has nice stationarity properties, it can "distinguish between simultaneous arrivals within a batch of arrivals", and is (for the present time change set-up) an equivalence of a similar distribution in Brandt et al. [3]. It follows immediately that $P^{0}=P_{\Phi} \theta_{0}^{-1}=$ $\bar{P}_{\Phi} \theta_{0}^{-1}$. From this result, (3.2) and (3.14), we obtain immediately that

$$
\bar{P}_{\Phi}(A)=E\left(\frac{\Phi\left\{T_{0}\right\}}{\left(T_{1}-T_{0}\right) \bar{\Phi}} 1_{A} \circ \eta_{[\sigma]}\right) \text { and } P(A)=\bar{E}_{\Phi}\left(\frac{\bar{\Phi}}{\Phi\{0\}} \int_{0}^{T_{1}} 1_{A} \circ \theta_{s}\right) d s
$$

where $[\sigma]$ is the largest number in $\left[D_{0}, \sigma\right]$ which is integer-distanced from $D_{0}$. The left-hand side of (3.17) is of special interest. It ensures that the time-stationary distribution $P$ can be transformed into the distribution $\bar{P}_{\Phi}$ by a two-step procedure consisting of a change of measure and the application of the shift $\eta_{[\sigma]^{\circ}}$. These observations are not only of interest for getting a good understanding of $\left.P_{\Phi} \sigma\right]^{\circ}$, but also for simulation purposes. See Section 5.

We complete this section with a short treatment of the case $p=0$, i.e., with $\Lambda$ being continuous, but $\Lambda$ having constant pairs. Thus, $p^{\prime}:=P\left(\Lambda^{\prime}\{0\}>0\right)$ is positive. In [9], it is noticed that $P_{\Lambda}$ and $P$ are dual in the sense that the DPD of $P_{\Lambda}$ w.r.t. $\Lambda^{\prime}$ is equal to $P$. From the arguments in Section 4 of this reference, it follows immediately that the results in the present research remain valid if $\Lambda$ is replaced by $\Lambda^{\prime}, P$ by $P_{\Lambda}, P_{\Lambda}$ by $P, \theta$ by $\eta$, and $\eta$ by $\theta$. In this context, we also replace functions of $\Lambda$ by functions of $\Lambda^{\prime}$, attaching a prime to the notations. For instance, $T_{i}^{\prime}$ is the $i$ th occurrence of $\Lambda^{\prime}, D_{i}^{\prime}$ : $=\Lambda^{\prime}\left(T_{i}^{\prime}-\right), S_{i}^{\prime}:=\Lambda^{\prime}\left(T_{i}^{\prime}\right)$, and $\sigma^{\prime}:=D_{0}^{\prime}+T_{1}^{\prime}\left(S_{0}^{\prime}-D_{0}^{\prime}\right) /\left(T_{1}^{\prime}-T_{0}^{\prime}\right)$. 
Set $P_{1}(B):=P\left(B \mid \Lambda^{\prime}\{0\}>0\right)$. With these considerations in mind, we can (for instance) deduce from Theorem $3.1(a),(3.2)$ and (3.8) that

$$
\begin{array}{ll}
P_{\Lambda}\left(\theta_{\sigma^{\prime}}^{-1} A\right) & =p^{\prime} E_{1}\left(\gamma_{0}^{\prime} 1_{A}\right), \\
P(A) & =\frac{1}{p^{\prime}} E_{\Lambda}\left(\frac{1}{\gamma_{0}^{\prime}} 1_{A} \circ \theta_{\sigma^{\prime}}\right) \\
P_{\Lambda}(A) & =p^{\prime} E_{1}\left(\gamma_{0}^{\prime} 1_{A} \circ \eta_{V^{\prime}}\right) ; A \in \mathscr{F} .
\end{array}
$$

\section{Local Characterization of PDs}

The PD of a point process with only single arrivals is often described as a conditional distribution, given an arrival at the origin. This intuitive interpretation of the PD is justified by the well-known local characterization theorem. In the present section, we will consider local aspects of Palm theory for general RTCs. At first, we derive a result which is Dobrushin's lemma in case of an ergodic PP. Then we prove results which give local characterizations of the conditional DPDs and OPDs. In the case of a bulk arrival process, this leads to a heuristic interpretation of $\bar{P}_{\Phi}$ as a conditional distribution.

Let $F$ be the $P$-distribution function of $X=U \gamma_{0}=T_{1} \bar{\Lambda} /\left(S_{0}-D_{0}\right)$ (cf. (2.11)) and suppose that $p>0$. By Lemma $2.2(d)$ and Theorem $3.1(a)$ we have, for all $t \geq 0$ :

$$
\begin{aligned}
F(t)=P(X & \leq t)=E\left(\frac{t}{\gamma_{0}} \wedge 1\right)=p E_{1 \Lambda}\left(t \wedge \gamma_{0}\right) \\
& =p \int_{0}^{t} P_{1 \Lambda}\left(\gamma_{0}>s\right) d s
\end{aligned}
$$

Hence, $F$ is differentiable with derivative $F^{\prime}(t)=p P_{1 \Lambda}\left(\gamma_{0}>t\right)$. Again by Theorem $3.1(a)$, we obtain:

$$
F(t)=P\left(\gamma_{0} \leq t\right)+p t P_{1 \Lambda}\left(\gamma_{0}>t\right), \quad t \geq 0
$$

From (4.2), it follows immediately that $F(t) \geq p t P_{1 \Lambda}\left(\gamma_{0}>t\right)$ and that

$$
F(t)=p E_{1 \Lambda}\left(\gamma_{0} 1_{\left(\gamma_{0} \leq t\right)}\right)-p t P_{1 \Lambda}\left(\gamma_{0} \leq t\right)+p t \leq 0+p t
$$

Consequently,

$$
\lim _{t \downarrow 0} \frac{1}{t} F(t)=p \text { if } p>0 .
$$

If $\Lambda=\Phi$ is a simple, ergodic PP with intensity $\lambda$, then (4.3) can be rewritten as $P(\Phi(0, t]>0)=\lambda t+o(t)$, as $t \rightarrow 0$. This is exactly Dobrushin's lemma; see, e.g. p 39 in $[2]$.

If both $p$ and $q$ are positive, we can derive similar results for the $P$-distribution function $G$ of $Y$ (cf. (2.11)): just replace $p$ and $\gamma_{0}$ in (4.1)-(4.3) by $q$ and $\delta_{0}$. The same holds for the $P_{1 \Lambda^{-}}$and the $P_{2 \Lambda^{-}}$-distribution functions $H$ and $J$ of

$$
Z_{1}:=U^{\prime} \frac{1}{\gamma_{0}}=\frac{S_{0}}{T_{1}-T_{0}} \frac{1}{\bar{\Lambda}} \text { and } Z_{2}:=U^{\prime \prime} \frac{1}{\delta_{0}}=\frac{D_{1}^{*}}{T_{1}^{*}-T_{0}^{*}} \frac{1}{\bar{\Lambda}},
$$


respectively. Just replace $\left(p, P_{1 \Lambda}, \gamma_{0}, P\right)$ by $\left(1 / p, P, 1 / \gamma_{0}, P_{1 \Lambda}\right),\left(1 / q, P, 1 / \delta_{0}, P_{2 \Lambda}\right)$, respectively. (For the proofs, we use Lemma 2.2, parts $(b)$ and $(c)$.)

Recall that $U=T_{1} /\left(T_{1}-T_{0}\right)$ and $X=T_{1} \bar{\Lambda} /\left(S_{0}-D_{0}\right)$. For a simple and ergodic PP, local characterization expresses that $P^{0}(A)$ can be approximated by the conditional probabilities $P\left(\theta_{T_{0}}^{-1} A \mid T_{1} \leq 1 / n\right)$, as $n \rightarrow \infty$, leading to the well-known intuitive interpretation of $P^{0}$ as a conditional distribution under $P$. (See, e.g., Theorem 10 in [11] for a uniform version.) In view of this observation, one might expect that, in the general RTC case, the conditional probabilities $P\left(\eta_{\sigma}^{-1} A \mid X \leq 1 / n\right)$ tend to $P_{1 \Lambda}(A)$ as $n \rightarrow \infty$. Unfortunately, this is not the case. Note in this context that

$$
\left(S_{0}-D_{0}\right) \circ \eta_{\sigma}=S_{0}-D_{0} \text { and } D_{0} \circ \eta_{\sigma}=-U\left(S_{0}-D_{0}\right)
$$

With $U_{0}^{\prime}:=-D_{0} /\left(S_{0}-D_{0}\right)=1-U^{\prime}$, we obtain by Theorem 3.1 and (4.1) that

$$
\begin{gathered}
P\left(\eta_{\sigma}^{-1} A \mid X \leq \frac{1}{n}\right)=\frac{E\left(1_{A} \circ \eta_{\sigma} \cdot 1\left(U_{0}^{\prime} \leq \frac{1 / n}{\gamma_{0}}\right)\right.}{E\left(1 \wedge \frac{1 / n}{\gamma_{0}}\right)} \\
=\frac{E_{1 \Lambda}\left(1_{A} \gamma_{0} 1_{\left(U_{0}^{\prime} \leq \frac{1 / n}{\gamma_{0}}\right)}\right)}{E_{1 \Lambda}\left(\gamma_{0} \wedge \frac{1}{n}\right)} .
\end{gathered}
$$

The choice $A=\left(U_{0}^{\prime} \leq 1 / 2\right)$ makes it clear that, in general, (4.5) will not tend to $P_{1 \Lambda}(A)$. Studying (4.5) in more detail yields that a reason might be that, in spite of Lemma $2.1(b)$, the random variable $U$ in $X=U \gamma_{0}$ is $P$-dependent of $\Lambda$. Let $\widetilde{U}_{1}$ be another uniformly $(0,1)$ distributed random variable on $(\Omega, \mathcal{F}, P)$ but $\widetilde{U}_{1}$ is assumed to be $P$-independent of $\eta_{\sigma}^{-1} \mathscr{F}$. (Here we assume that $\Omega$ is rich enough to support such a variable.) By conditioning on the $\sigma$-field generated by $\gamma_{0}$, we obtain that

$$
P\left(\eta_{\sigma}^{-1} A \mid \tilde{U}_{1} \gamma_{0} \leq \frac{1}{n}\right)=\frac{E\left(1_{A} \circ \eta_{\sigma} \cdot\left(1 \wedge \frac{1 / n}{\gamma_{0}}\right)\right)}{E\left(1 \wedge \frac{1 / n}{\gamma_{0}}\right)}=: P_{n}(A)
$$

A similar relation can be obtained in terms of $\tau$ and $\delta_{0}$. Suppose that $\widetilde{U}_{2}$ is a uniform $(0,1)$ random variable which is $P$-independent of $\eta_{\tau}^{-1} \sigma$. Then we have

$$
P\left(\eta_{\tau}^{-1} A \mid \tilde{U}_{2} \delta_{0} \leq \frac{1}{n}\right)=\frac{E\left(1_{A} \circ \eta_{\tau} \cdot\left(1 \wedge \frac{1 / n}{\delta_{0}}\right)\right)}{E\left(1 \wedge \frac{1 / n}{\delta_{0}}\right)}=: Q_{n}(A)
$$

After all, $P_{n}$ and $Q_{n}$ do not depend on $\widetilde{U}_{1}$ and $\widetilde{U}_{2}$, respectively. These uniform $(0,1)$ random variables are only used to write $P_{n}$ and $Q_{n}$ as conditional probability measures.

The next theorem expresses local characterizations of the conditional DPDs. For the proof, we need a well-known general result. Let $Q_{1}, Q_{2}$ and $Q$ be probability measures on a common measurable space. Suppose that the null sets of $Q$ are also null sets of both $Q_{1}$ and $Q_{2}$. Let $h_{i}$ be the density of $Q_{i}$ w.r.t. $Q(i=1,2)$. Then, 


$$
2_{A}^{\sup }\left|Q_{1}(A)-Q_{2}(A)\right|=\int\left|h_{1}-h_{2}\right| d Q .
$$

(See, e.g. [12] for a short proof.)

Theorem 4.1: Suppose that the RTC $\Lambda$ satisfies (i)-(iii). Then

$$
\begin{aligned}
& \sup _{A \in \mathcal{F}}\left|P_{n}(A)-P_{1 \Lambda}(A)\right|=1-\frac{F\left(F\left(\frac{1}{n}\right) / p\right)}{F\left(\frac{1}{n}\right)} \rightarrow 0 \\
& \sup _{A \in \mathcal{F}}\left|Q_{n}(A)-P_{2 \Lambda}(A)\right|=1-\frac{G\left(G\left(\frac{1}{n}\right) / q\right)}{G\left(\frac{1}{n}\right)} \rightarrow 0
\end{aligned}
$$

as $n \rightarrow \infty$, provided that $p$ is positive and both $p$ and $q$ are positive, respectively.

Proof: Set $h(n)=F(1 / n) / p$. By Theorem 3.1, it follows immediately that the null sets of $P_{1 \Lambda}$ are also null sets of $P_{n}$. By the result preceding this theorem, the supremum in (4.8) is equal to $E_{1 \Lambda}\left|\gamma_{0} \wedge(1 / n)-h(n)\right| /(2 h(n))$. This in turn can be written as a summation of $P_{1 \Lambda^{-}}$and $P$-probabilities by partitioning $\Omega$ into the parts $\left(\gamma_{0} \leq h(n)\right),\left(h(n)<\gamma_{0} \leq 1 / n\right)$, and $\left(\gamma_{0}>1 / n\right)$, and applying Theorem $3.1(a)$. The equality in (4.8) follows by properly applying (4.2). The convergence in (4.8) is a consequence of (4.3) when replacing $t$ by $F(1 / n) / p$. The proof of (4.9) is similar.

Remark 4.1: Since $1_{A} \circ \theta_{0} \circ \eta_{\sigma}=1_{A} \circ \theta_{T_{0}}$ and since (cf. Lemma 2.1) $U=$ $T_{1} /\left(T_{1}-T_{0}\right)$ is indeed $P$-independent of $\theta_{T_{0}}^{-1} \mathscr{F}$, it follows immediately that

$$
\sup _{A \in \mathcal{F}}\left|P\left(\theta_{T_{0}}^{-1} A \mid X \leq \frac{1}{n}\right)-P_{1 \Lambda}\left(\theta_{0}^{-1} A\right)\right| \rightarrow 0
$$

as $n \rightarrow \infty$. Since $P_{1 \Lambda} \theta_{0}^{-1}$ is just the conditional OPD (cf. (3.13)), (4.10) is for the present RTC-setting the generalization of Theorem 10 in [11]. See also [17] for a process with cycles setting. If $p=1$ (i.e., if $\Lambda$ is a pure jump process), then (4.10) expresses a local characterization of the OPD of $\Lambda$. It is well-known in the case of simple PPs.

Unfortunately, we had to choose an abstract uniformly $(0,1)$ distributed random variable $\tilde{U}_{1}$ - that is, not a concrete function of $\Lambda$ - to arrange the desired independence and the local characterization in (4.8); recall the arguments following (4.5). As a corollary, for a general RTC, (4.8) does not lead to a nice heuristical interpretation of $P_{1 \Lambda}$. However, in special cases, $\widetilde{U}_{1}$ can be adjusted. We investigate th local characterization of $\bar{P}_{\Phi}$, the distribution of detailed Palm type which has nice properties in case the RTC is a not necessarily simple point process $\Phi$; see Example 3.1. As usual, we assume that within a batch, the arrivals are ordered. Although $\bar{P}_{\Phi}=P_{\Phi} \eta_{\alpha}^{-1}$, we can't use an approach as in Remark 4.1 since $\eta_{\alpha} \circ \eta_{\sigma}=\eta_{[\sigma]}$ depends on $U$, and $U$ and $\eta_{[\sigma]}^{-1} \sigma$ are $P$-dependent It looks like we really need an abstract uniform $(0,1)$ random variable as $\widetilde{U}_{1}$ in $(4.6)$. However, in the present PP case, we still can choose $\widetilde{U}_{1}$ as a function of $\Phi$. Recall that $T_{i-1}$ is the $(i-1)$ th jump time, and that $S_{i-1}-D_{i-1}$ is the corresponding jump size (which is now integer-valued), $i \in \mathbb{Z}$. We partition the interval $\left[T_{i-1}, T_{i}\right)$ into $S_{i-1}-D_{i-1}$ subintervals of equal lengths by defining the intermediate times

$$
T_{i-1, j}:=T_{i-1}+j \frac{T_{i}-T_{i-1}}{S_{i-1}-D_{i-1}} ; j=0, \ldots, S_{i-1}-D_{i-1} .
$$

For $s$ between $T_{i-1, j-1}$ and $T_{i-1, j}$, we have

$$
\eta_{\alpha} \circ \eta_{\sigma} \circ \theta_{s}=\eta_{S_{i-1}-j} ; j=1, \ldots, S_{i-1}-D_{i-1} .
$$


Let $\widetilde{T}_{1}$ and $\widetilde{T}_{0}$, respectively, be the smallest positive and the largest non-positive intermediate times in $\left[T_{0}, T_{1}\right]$, and set $\widetilde{U}:=\widetilde{T}_{1} /\left(\widetilde{T}_{1}-\widetilde{T}_{0}\right)$. Note that $\widetilde{U}=$ $\widetilde{T}_{1}\left(S_{0}-D_{0}\right) /\left(T_{1}-T_{0}\right)$ and that $\widetilde{U} \gamma_{0}=\widetilde{T}_{1} \bar{\Phi}$. Part $(b)$ below expresses local characterization of $\bar{P}_{\Phi}$.

Theorem 4.2: Let $\Phi$ be a (possibly non-simple) PP, which satisfies assumptions (i)-(iii) and let $[\sigma]$ be the largest number in $\left[D_{0}, \sigma\right]$, which is integer-distanced from $D_{0}$. Then:

(a) $\eta_{[\sigma]}^{-1} \sigma$ and $\tilde{U}$ are independent under $P$, while $\widetilde{U}$ is uniformly $(0,1)$

(b) $\sup _{A \in \mathcal{F}}\left|P\left(\eta_{[\sigma]}^{-1} A \mid \widetilde{T}_{1} \bar{\Phi} \leq \frac{1}{n}\right)-\bar{P}_{\Phi}(A)\right| \rightarrow 0$.

Proof: $(a)$ Let $f$ and $g$ be suitably measurable functions. Denote the number of $T_{i} \mathbf{s}$ in $(0, t]$ by $N(t)$. By $(2.8)$, and by partitioning $(0, t]$ into the subintervals $\left(T_{i-1}, T_{i}\right.$ ] and partitioning these intervals in turn into the subintervals constituted by the intermediate times, we obtain that

$$
\begin{gathered}
E\left(f(\tilde{U}) g \circ \eta_{\alpha} \circ \eta_{\sigma}\right) \\
=\lim _{t \rightarrow \infty} E_{\Phi}\left(\frac{1}{t} \sum_{i=1}^{N(t)} \sum_{j=1}^{S_{i-1}-D_{i-1}} \int_{T_{i-1, j-1}}^{T_{i-1, j}} f\left(\frac{T_{i-1, j}-s}{T_{i-1, j}-T_{i-1, j-1}}\right) \cdot g \circ \eta_{S_{i-1}-j} d s\right) \\
=\int_{0}^{1} f(u) d u \cdot \lim _{t \rightarrow \infty} E_{\Phi}\left(\frac{1}{t} \sum_{i=1}^{N(t)} \frac{T_{i}-T_{i-1}}{S_{i-1}-D_{i-1}} \sum_{j=1}^{S_{i-1}} g \circ \eta_{S_{i-1}-j}\right) \\
=\int_{0}^{1} f(u) d u \cdot E\left(g \circ \eta_{\alpha} \circ \eta_{\sigma}\right) .
\end{gathered}
$$

Here the third equality follows for general $f$ and $g$, by applying the second equality for $f \equiv 1$.

(b) This follows from (4.6) and (4.8) replacing $A$ by $\eta_{\alpha}^{-1} A, \widetilde{U}_{1}$ by $\widetilde{U}$, and $\mathscr{F}$ by $\eta_{\alpha}^{-1}{ }_{\mathcal{F}}$

Remark 4.2: For a heuristic interpretation of part $(b)$ of the theorem, recall that working under $P$ means that the origin is uniformly situated in the interval $\left(T_{0}, T_{1}\right)$; cf. Lemma $2.1(b)$ in this paper and Remark 4.1 in [9]. Hence, letting $t$ tend to zero, with $\widetilde{T}_{1} \leq t$, implies that in equilibrium the origin falls in an intermediate time, $M$ units of $\left(T_{1}-T_{0}\right) /\left(S_{0}-D_{0}\right)$ away from $T_{1}$. Here the integer-valued random variable $M$ is (conditionally) uniformly distributed in $\left\{1, \ldots, S_{0}-D_{0}\right\}$. But in this ultimate situation, $\sigma$ equals $D_{0}+M$. Hence, heuristically, $\bar{P}_{\Phi}$ arises from $P$ by conditioning on the origin falling in an intermediate time, and - with $M$ intermediate times on its right in $\left(0, T_{1}\right]$ - moving the origin to $\left(T_{0}, D_{0}+M\right)$.

Obviously, transitions from $P$ to $P_{1 \Lambda}$ and to $P_{2 \Lambda}$ (as described above) are important for getting a good understanding of the DPD of an RTC. Finally, we consider transitions the other way round: from $P_{1 \Lambda}$ to $P$ and from $P_{2 \Lambda}$ to $P$. Since $\theta_{V}$ and $\theta_{W}$ are in a sense - see (3.4) and (3.5) - the inverse shifts of $\eta_{\sigma}$ and $\eta_{\tau}$, respectively, the following results are obvious:

$$
P_{1 \Lambda}\left(\theta_{V}^{-1} A \mid \frac{\widetilde{U}_{3}}{\gamma_{0}} \leq \frac{1}{n}\right)=\frac{E_{1 \Lambda}\left(1_{A} \circ \theta_{V} \cdot\left(1 \wedge \frac{\gamma_{0}}{n}\right)\right)}{E_{1 \Lambda}\left(1 \wedge \frac{\gamma_{0}}{n}\right)}=: R_{n}(A)
$$




$$
P_{2 \Lambda}\left(\theta_{W}^{-1} A \mid \frac{\widetilde{U}_{4}}{\delta_{0}} \leq \frac{1}{n}\right)=\frac{E_{2 \Lambda}\left(1_{A} \circ \theta_{W} \cdot\left(1 \wedge \frac{\delta_{0}}{n}\right)\right)}{E_{2 \Lambda}\left(1 \wedge \frac{\delta_{0}}{n}\right)}=: S_{n}(A)
$$

$A \in \mathcal{F}$. Here $\widetilde{U}_{3}$ and $\widetilde{U}_{4}$ are uniformly $(0,1)$ distributed random variables, $P_{1 \Lambda^{-i n d e-}}$ pendent of $\theta_{V}^{-1}{ }_{\mathscr{F}}$ and $P_{2 \Lambda}$-independent of $\theta_{W}^{-1} \mathscr{F}$, respectively. By using the distribution functions $H$ and $J$ of $Z_{1}$ and $Z_{2}$ (see (4.4)), it can be proven that $R_{n}(A)$ and $S_{n}(A)$ tend to $P(A)$, uniformly over $A \in \mathcal{F}$.

\section{Simulation Applications}

$P$ describes the random behavior of the RTC as it is seen from a randomly chosen time-point (on the horizontal axis), $P_{\Lambda}$ as it is seen from a randomly chosen level (on the vertical axis). Under $P$, a typical realization will have its origin in a continuous part of the graph, but it might be constant (and hence equal to zero) on a small interval $(0, \epsilon)$. Under $P_{\Lambda}$, there are two types of typical realizations. For the first type, the origin is in a jump part; for the second, it lies in a continuous part but the time change is strictly increasing on $(0, \epsilon)$ for $\epsilon$ small enough. In the present section, we will in particular consider procedures for generating these two types of realizations when starting with realizations typical under $P$. The procedures originate from Asmussen et al. [1] and [17], and they are of special interest whenever it is easier (or less time-consuming) to obtain realizations of the RTC from one viewpoint than it is from another. In the case of a PP with multiple arrivals, the main procedure generates a version of the PP as seen through the eyes of an arbitrarily chosen arrival within a batch of simultaneous arrivals at the origin.

At first we generate a version of the RTC under $P_{1 \Lambda}$, starting with independent versions under $P$. Let $p$ be positive. For a version $\Lambda_{n}$ of $\Lambda$, we will occasionally write $T_{j}^{(n)}, T_{j}^{*(n)}($ for $j \in \mathbb{Z}), S_{0}^{(n)}, D_{0}^{(n)}, D_{1}^{(n)}, S_{0}^{*(n)}, D_{0}^{*(n)}, D_{1}^{*(n)}, \gamma_{0}^{(n)}, \delta_{0}^{(n)}, \sigma_{n}$, $\tau_{n}, V_{n}$ and $W_{n}$, in accordance with the corresponding definitions in Sections 2-4. For a positive and fixed constant $b$, the recursive procedure (for $n \in \mathbb{N}$ ) with the steps

(1) generate a version $\Lambda_{n}$ of the RTC under $P$, independent of $\Lambda_{1}, \ldots, \Lambda_{n-1}$,

(2) $\quad$ set $\Lambda_{0 n}:=\Lambda_{n} \circ \eta_{\sigma_{n}}$

(3) generate under $P$, a uniform $(0,1)$ observation $U_{n}$, independent of $U_{1}, \ldots, U_{n-1}$ and $\Lambda_{01}, \ldots, \Lambda_{0 n}$,

(4) stop the procedure as soon as $U_{n} \leq b / \gamma_{0}^{(n)}$ and set $M:=\min \left\{n \in \mathbb{N}: U_{n} \leq\right.$ $\left.b / \gamma_{0}^{(n)}\right\}$

leads to a version $\Lambda_{0 M}$. For a measurable function $f$, we obtain that

Note that

$$
\begin{gathered}
E\left(f\left(\Lambda_{0 M}\right)\right)=\sum_{n=1}^{\infty} E\left(f\left(\Lambda_{0 n}\right) 1_{(M=n)}\right) \\
=\sum_{n=1}^{\infty} E\left(f\left(\Lambda_{0 n}\right) 1_{\left(U_{n} \leq b / \gamma_{0}^{(n)}\right)}\right) P(M>n-1) \\
=E\left(f\left(\Lambda_{01}\right) 1_{\left(U_{1} \leq b / \gamma_{0}^{(1)}\right)}\right) \cdot E(M) .
\end{gathered}
$$

$$
E(M)=\sum_{n=0}^{\infty}\left(P\left(U_{1}>b / \gamma_{0}^{(1)}\right)^{n}=1 / P\left(U_{1} \leq b / \gamma_{0}^{(1)}\right),\right.
$$




$$
\begin{gathered}
E\left(f\left(\Lambda_{0 M}\right)\right)=\frac{E\left(f\left(\Lambda \circ \eta_{\sigma}\right) \cdot\left(1 \wedge \frac{b}{\gamma_{0}}\right)\right)}{E\left(1 \wedge \frac{b}{\gamma_{0}}\right)} \\
=\frac{E_{1 \Lambda}\left(f(\Lambda)\left(\gamma_{0} \wedge b\right)\right)}{E_{1 \Lambda}\left(\gamma_{0} \wedge b\right)} .
\end{gathered}
$$

See Theorem 3.1 ( $a$ ) for the last equality. By (4.6) and (4.8), the $P$-distribution of the version $\Lambda_{0 M}$ of the RTC tends to the $P_{1 \Lambda}$-distribution of the RTC as $b$ tends to zero from above. If $P\left(\gamma_{0}>b\right)=1$, it follows immediately that $E\left(f\left(\Lambda_{0 M}\right)\right)=$ $E_{1 \Lambda}(f(\Lambda))$. The following intuitive statement declares what is going on (renaming $\Lambda_{0 M}$ as $\left.\Lambda_{0 M}^{(1)}\right)$ :

$\Lambda_{0 M}^{(1)}$ is a typical version of the RTC as seen from a level chosen at random in its jump parts,

provided that, with probability one, $\gamma_{0}$ is larger than a positive constant. Note also that, under this hypothesis, the right-continuous inverse of the version generated by this procedure is just a version of $\Lambda^{\prime}$ as seen under $P_{\Lambda}$. For instance, the inverse of the pure jump process $\Lambda$ with jump sizes $X_{i}$ and $T_{i}-T_{i-1}=1$ is a simple point process. So, the above procedure can be used to transform realizations of a $P$-stationary sequence $\left\{X_{i}\right\}$ into a realization of a time-stationary point process with intensity $1 / E X_{i}$

In the case that $\Lambda=\Phi$ is a PP with multiple arrivals, the above procedure can easily be adapted to generate a version of the PP as seen under $\bar{P}_{\Phi}$. That is, to generate a version as seen through the eyes of an arbitrarily chosen arriving customer within a batch of simultaneous arrivals in the origin. Just replace step 2 by $\Lambda_{0 n}$ : = $\Lambda_{n} \circ \eta_{\alpha_{n}} \circ \eta_{\sigma_{n}}=\Lambda_{n} \circ \eta_{\left[\sigma_{n}\right]}$, replace all $\Lambda \mathrm{s}$ by $\Phi \mathrm{s}$, and recall that in this case, $P_{1 \Lambda} \eta_{\alpha}^{-1}$ is just $\bar{P}_{\Phi}$.

If both $p$ and $q$ are positive, a similar procedure leads to a typical version of the RTC as seen under $P_{2 \Lambda}$ : just replace $\left(\sigma_{n}, \gamma_{0}^{(n)}\right)$ by $\left(\tau_{n}, \delta_{0}^{(n)}\right)$. By Theorems $3.1(b)$ and (4.9), we obtain that the $P$-distribution of the version $\Lambda_{0 M}^{(2)}$, which follows from the adapted procedure, tends to the $P_{2 \Lambda}$-distribution of the RTC (as $\left.b \downarrow 0\right)$, and

$\Lambda_{O M}^{(2)}$ is a typical version of the RTC as seen from a level chosen at random in its continuous parts,

provided that, with probability one, $\delta_{0}$ is larger than a positive constant.

With (3.8), (3.9), (4.13) and (4.14) in mind, we can also formulate procedures, which work the other way around. Starting with versions $\Lambda_{n}$ under $P_{1 \Lambda}$ or $P_{2 \Lambda}$, we just have to replace $\left(b, P, \eta_{\sigma_{n}}, \gamma_{0}^{(n)}\right)$ in the procedure by $\left(1 / b, P_{1 \Lambda}, \theta_{V_{n}}, 1 / \gamma_{0}^{(n)}\right)$ or $\left(1 / b, P_{2 \Lambda}, \theta_{W_{n}}, 1 / \delta_{0}^{(n)}\right)$. The respective versions $\Lambda_{0 M}$ are both versions of the RTC under $P$.

Finally, we note that, under a similar boundedness condition, (3.11) (or (3.12)) leads to a procedure to derive an observation under $P_{1 \Lambda}\left(\right.$ or $\left.P_{2 \Lambda}\right)$, when starting with observations of the RTC under $P_{2 \Lambda}$ (or $P_{1 \Lambda}$ ). Here, we have to replace $\eta_{\sigma_{n}}$ by $\eta_{\sigma_{n}} \circ \theta_{W_{n}}\left(\right.$ or $\left.\eta_{\tau_{n}} \circ \theta_{V_{n}}\right)$. 


\section{Acknowledgement}

The author would like to thank the referee and the editor for their helpful comments.

\section{References}

[1] Asmussen, S., Glynn, P. and Thorisson, H., Stationarity detection in the initial transient problem, ACM Trans. Modeling Comput. Simul. 2 (1992), 130-157.

[2] Baccelli, F. and Brémaud, P., Elements of Queueing Theory, Springer, New York 1994.

[3] Brandt, A., Franken, P. and Lisek, B., Stationary Stochastic Models, Wiley, New York 1990.

[4] Franken, P., König, D., Arndt, U. and Schmidt, V., Queues and Point Processes, Wiley, New York 1982.

[5] Geman, D. and Horowitz, J., Remarks on Palm measures, Ann. Inst. Henri Poincaré IX:3 (1973), 215-232.

[6] Mecke, J., Stationäre zufällige Masse auf lokalkompakten Abelschen Gruppen, Z. Wahrsch. verw. Geb. 9 (1967), 36-58.

[7] Miyazawa, M., Palm calculus for a process with a stationary random measure and its applications to fluid queues, Queueing Systems 17 (1994), 183-211.

[8] Miyazawa, M. and Schmidt, V., On level crossings of stochastic processes with stationary bounded variations and continuous decreasing components, Prob. and Math. Stat. 17 (1997), 79-93.

[9] Miyazawa, M., Nieuwenhuis, G. and Sigman, K., Palm theory for random time changes, J. of Appl. Math and Stoch. Anal. (2001), to appear.

[10] Nieuwenhuis, G., Equivalence of functional limit theorems for stationary point processes and their Palm distributions, Prob. Th. Rel. Fields 81 (1989), 593608.

[11] Nieuwenhuis, G., Bridging the gap between a stationary point process and its Palm distribution, Statistica Neerlandica 48:1 (1994), 37-62.

[12] Nieuwenhuis, G., Ergodicity conditions and Cesáro limit results for marked point processes, Stochastic Models 14:3 (1998), 681-714.

[13] Ryll-Nardzewski, C., Remarks on processes of calls, Proc. Fourth Berkeley Symp. Math. Stat. Prob. 2 (1961), 455-465.

[14] Schmidt, V. and Serfozo, R.F., Campbell's formula and applications to queueing, In: Advances in Queueing (ed. by J.H. Dshalalow), Chapter 8, CRC Press, Boca Raton, FL (1995), 225-242.

[15] Sigman, K., Stationary Marked Point Processes: An Intuitive Approach, Chapman and Hall, New York 1995.

[16] Slivnyak, I.M., Some properties of stationary flows of homogeneous random events, Teor. Veroyat. Primen. 7 (1961), 347-352. [Transl. in Theory Prob. Appl. 7 (1962), 336-341.]

[17] Thorisson, H., On time- and cycle-stationarity, Stoch. Proc. and Their Appl. 55 (1995), 183-209. 


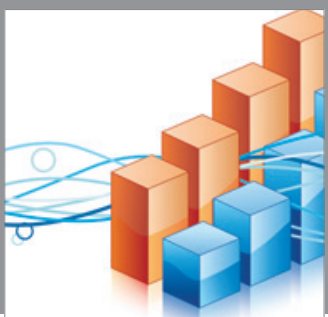

Advances in

Operations Research

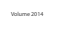

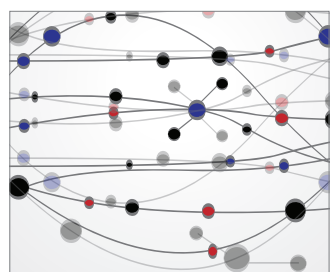

\section{The Scientific} World Journal
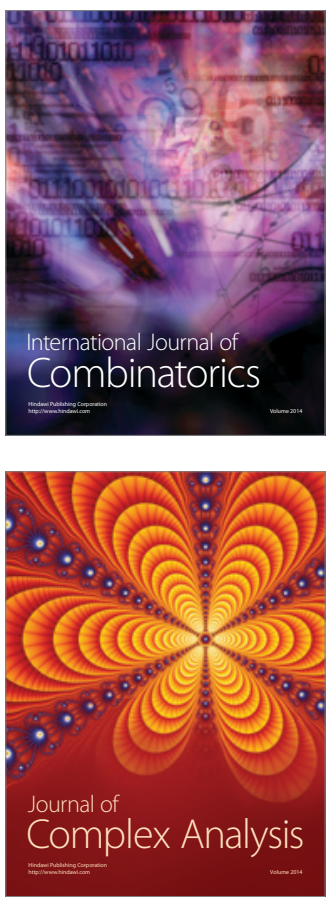

International Journal of

Mathematics and

Mathematical

Sciences
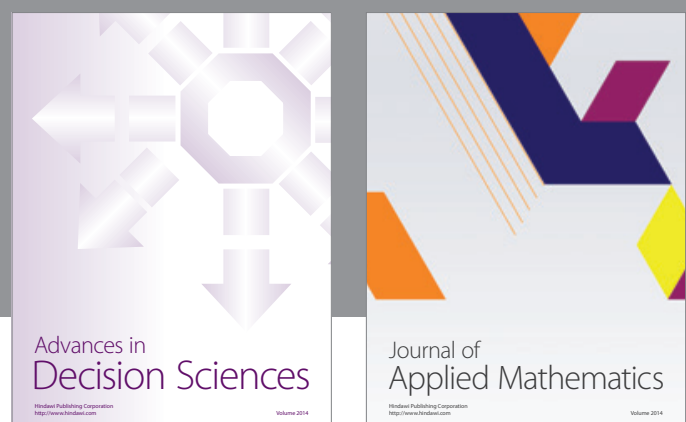

Journal of

Applied Mathematics
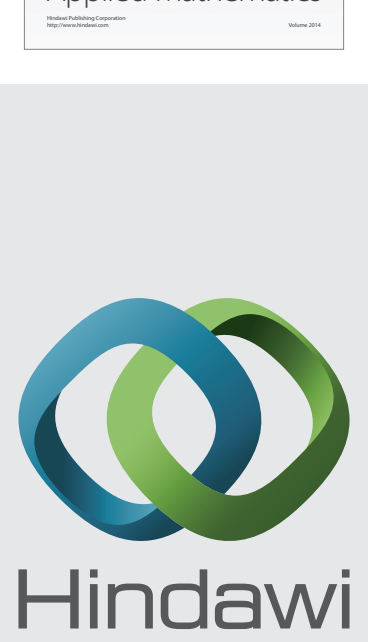

Submit your manuscripts at http://www.hindawi.com
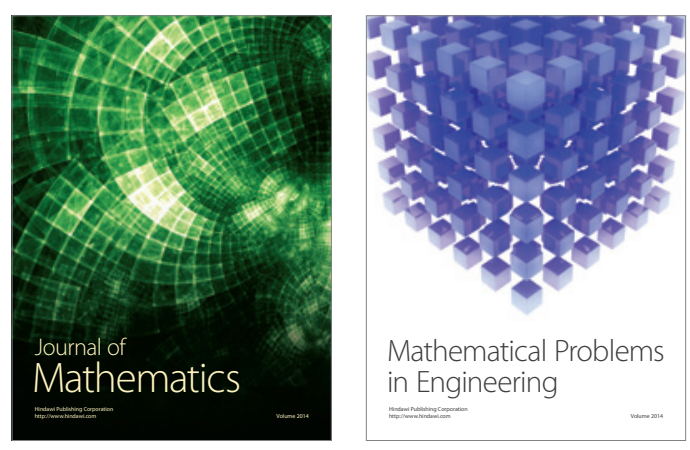

Mathematical Problems in Engineering
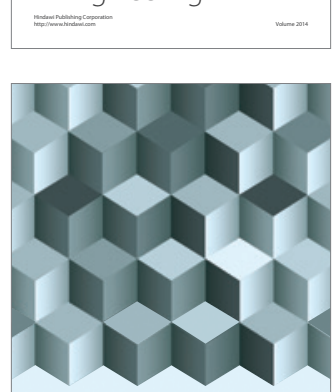

Journal of

Function Spaces
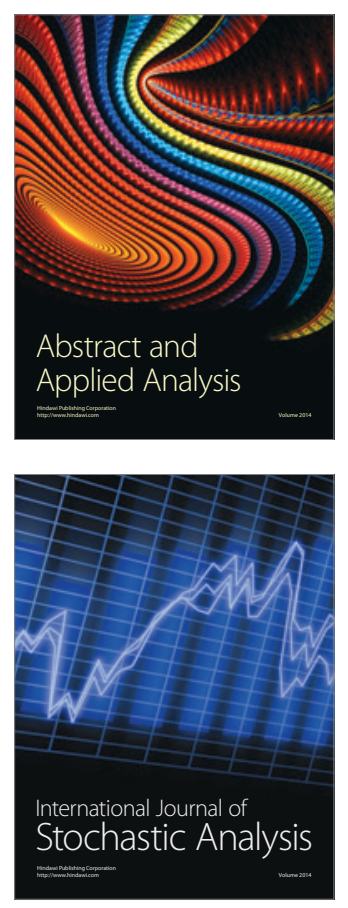

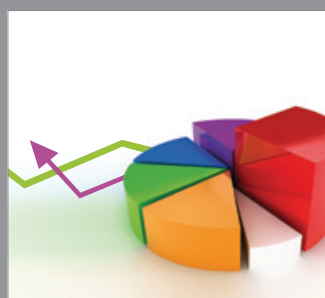

ournal of

Probability and Statistics

Promensencen
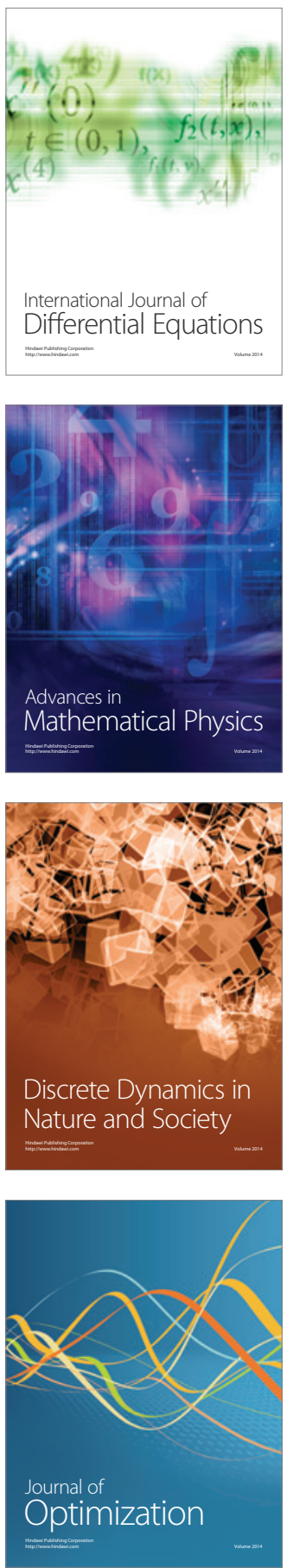